\title{
RESULTS OF A TOTAL AORTIC ARCH REPLACEMENT FOR AN ACUTE AORTIC ARCH DISSECTION
}

Takashi Hirotani, MD

Tadashi Kameda, MD

Takayuki Kumamoto, MD

Shogo Shirota, MD
Objectives: Recently, the immediate results of a surgical repair for an acute aortic arch dissection have dramatically improved. However, a total aortic arch replacement is recommended in a limited number of patients with an intimal tear located in the aortic arch. We have performed a total aortic arch replacement for all such patients with an acute aortic arch dissection since September 1995.

Methods: During the past 4 years, 27 consecutive patients who had an aortic arch dissection underwent a total aortic arch replacement. Twenty-five patients underwent an emergency operation. In 5 patients the intimal tear was located in the aortic arch, but in the rest of the patients, it was located in the ascending aorta or the proximal descending aorta. To obliterate any false channels, gelatin-resorcin-formol glue was used.

Results: The hospital mortality was $11 \%$, and no cerebral complications were observed. Postoperative aortography and computed tomography showed no evidence of any persisting false channels in 15 patients $(65 \%)$. During the follow-up period (ranging from 5 months to 4 years), two patients underwent a reoperation because of the recurrence of a dissection at the sinus of Valsalva. All patients, except for one who died after a reoperation, are still alive and free from any serious events at this writing.

Conclusions: Resecting both the ascending and transverse aorta, irrespective of whether the intimal tear is located in the aortic arch, may be an acceptable alternative at experienced centers because of its low mortality and good midterm results. (J Thorac Cardiovasc Surg 2000;120:686-91)
It has been clearly established that an acute type A aortic dissection requires emergency surgical intervention. Recently, the immediate results of a surgical repair for this disease have dramatically improved. Therefore, in addition to saving the patient, the prevention of late complications and deaths has now become the focus of modern surgery. The extent of resections has been expanded to realize this objective, and several recent

From the Department of Cardiovascular Surgery, Tokyo Saiseikai Central Hospital, Tokyo, Japan.

Received for publication Dec 1, 1999; revisions requested Feb 28, 2000; revisions received June 20, 2000; accepted for publication June 20, 2000.

Address for reprints: Takashi Hirotani, MD, Department of Cardiovascular Surgery, Tokyo Saiseikai Central Hospital, 1-714 Mita, Minato-ku, Tokyo 108-0073, Japan (E-mail: hero.takashi@nifty.ne.jp).

Copyright (c) 2000 by The American Association for Thoracic Surgery

$0022-5223 / 2000 \$ 12.00+0 \quad \mathbf{1 2 / 1 / 1 0 9 5 1 6}$

doi:10.1067/mtc.2000.109516 studies $^{1-3}$ have proposed that a simultaneous aortic arch and ascending aortic repair could be performed with both an acceptable mortality and better late results than a previous limited repair. However, in most reports ${ }^{4-6}$ such aggressive procedures are only recommended in specific settings. In Japan, use of gelatin-resorcin-formol (GRF) glue (Cardial, Saint-Etienne, France) has been allowed since September 1995. In addition, in a previous study ${ }^{7}$ we analyzed and reported the effects of our procedures for brain protection by using large doses of thiopental under profound hypothermic conditions. To improve the long-term results of operations for acute aortic arch dissection, our surgical strategy was determined as described below. Irrespective of whether the intimal tear is located in the aortic arch, a simultaneous replacement of the ascending and transverse aorta with the prosthesis is routinely performed for all patients whose aortic arch is injured by the dissection.

Our cardiovascular center was organized in 1992, and the number of patients who underwent the previous 
conservative strategy (ie, an ascending aortic repair alone or hemi-arch repair) was very small. Therefore, the results of our aggressive approach could not be compared with those of our previous repair.

Here we describe the early and midterm outcomes of this procedure while comparing our findings with those of a more conservative repair, as reported in the literature.

\section{Methods}

Patients. From September 1995 through August 1999, 27 consecutive patients (18 men and 9 women) underwent a graft replacement of the ascending and transverse aorta for an acute type A aortic dissection involving the aortic arch. All patients were managed according to our aggressive strategy. The age of the patients ranged from 39 to 79 years (mean, $63.3 \pm 9.2$ years). The preoperative complications related to the aortic dissection included shock, which was defined as a systolic blood pressure of less than $80 \mathrm{~mm} \mathrm{Hg}$ in 11 (41\%) patients; cardiac tamponade in $11(41 \%)$ patients; aortic regurgitation, which was observed on an echocardiogram to be more than a moderate degree in $11(41 \%)$ patients; transient cerebral ischemia in $6(22 \%)$ patients; renal ischemia, which was defined as a serum creatinine level of more than $2.0 \mathrm{~g} / \mathrm{dL}$ in $6(22 \%)$ patients; leg ischemia in $5(19 \%)$ patients; coronary ischemia in $3(11 \%)$ patients; paraparesis in $2(7 \%)$ patients; and mesenteric ischemia in $1(4 \%)$ patient. No patients demonstrated Marfan syndrome. The time of the initial dissection, as defined by the onset of chest pain, ranged from 4 hours to 7 days before the operation. Twenty-five $(93 \%)$ patients underwent an emergency operation within 24 hours of onset. In 2 patients the operation was not performed on an emergency basis because the false channel in the aortic arch was found to be obliterated by thrombi on computed tomography (CT) with contrast media.

All patients underwent preoperative CT to confirm the diagnosis, but $15(56 \%)$ patients did not undergo preoperative aortography because of their critical state. The intraoperative findings indicated that an intimal tear was located in the ascending aorta in $18(67 \%)$ patients, from the ascending aorta to the aortic arch in 1 patient, in the aortic arch in 4 patients, and in the proximal descending aorta in 3 patients. In 1 patient no intimal tear was found either during the operation or on postoperative aortography in either the ascending aorta, aortic arch, or descending aorta.

Surgical technique. According to our surgical strategy, all patients whose aortic dissection involved the aortic arch underwent a simultaneous replacement of the ascending aorta and the aortic arch. Both the nasopharyngeal and rectal temperatures were monitored, and electroencephalography was performed. A median sternotomy was performed in all patients. The femoral artery with a stronger pulsation and the right axillary artery were both cannulated to avoid any malperfusion caused by retrograde perfusion through the femoral artery. A venous drainage cannula was placed through the right atrium. The left side of the heart was vented through the right superior pulmonary vein. The patient was then started on cardiopulmonary bypass (CPB) and cooled systemically. After spontaneous ventricular fibrillation caused by cooling of the heart, the ascending aorta was crossclamped just proximal to the origin of the innominate artery. The aorta was opened longitudinally, and then the aortic root was inspected. Cold crystalloid cardioplegic solution was directly infused into the coronary ostia. The ascending aorta that was injured by the dissection was totally resected, or if the dissection progressed beyond the sinotubular junction, then the ascending aorta was transected circumferentially $1 \mathrm{~cm}$ above the coronary ostia, and the distal segment of the ascending aorta was resected. When the dissecting process remained in the sinus of Valsalva, the proximal aortic stump was reconstructed with continuous mattress sutures and circular Teflon collars, and GRF glue was applied to the false channel, as previously reported in detail. ${ }^{8}$ Attempts were made to preserve the aortic valve for all patients except for patients who had a pre-existing dilatation of the aortic sinuses. In 10 of 11 patients with acute aortic regurgitation, aortic valve resuspension was performed, whereas the remaining patient underwent a replacement of the aortic root with a composite valved conduit. Coronary artery bypass grafting was concomitantly performed in 1 patient whose right coronary ostium was severely compressed and stenosed by the dissection. Systemic cooling progressed until the attendant neurologists confirmed the total disappearance of any electroencephalographic activity. The pharmacologic combination of thiopental, nicardipine, and mannitol was used for brain protection before circulatory arrest, as previously reported. ${ }^{7}$ After CPB was discontinued, the aortic arch was opened, and the proximal descending aorta was transected circumferentially just distal to the ostium of the left subclavian artery or intimal tear in the proximal descending aorta if exposed. The aortic arch was routinely totally resected together with the proximal segments of the brachiocephalic vessels. The distal aortic stump was reconstructed in the same manner as the proximal stump. The false channels in the brachiocephalic vessels, when present, were also obliterated by using GRF glue. A prosthetic graft with 4 limbs, consisting of 3 limbs to reconstruct the brachiocephalic vessels and 1 limb to establish arterial access of CPB after aortic arch repair, was then anastomosed to the distal aortic stump, and thereafter the innominate and left common carotid arteries were reconstructed. Subsequently, the arch graft was crossclamped at the preinnominate area, and then CPB was resumed by using the limb for the arterial access of CPB. During rewarming, the arch graft was anastomosed to the proximal aortic stump, which had already been reconstructed, and then to the left subclavian artery. CPB was terminated when normothermia had been achieved.

CPB. The nasopharyngeal temperature reached $11^{\circ} \mathrm{C}$ to $22^{\circ} \mathrm{C}$ (mean, $14.7^{\circ} \mathrm{C} \pm 2.5^{\circ} \mathrm{C}$ ). The alpha-stat method of blood $\mathrm{pH}$ regulation was used during hypothermia. The duration of circulatory arrest ranged from 30 to 77 minutes and averaged $50.6 \pm 11.8$ minutes. 


\section{Results}

Operative mortality and morbidity. Three (11\%) of 27 patients died (5\% to $21 \%$ confidence limits) during hospitalization. The causes of death were graft-versus-host disease caused by intraoperative transfusion and sepsis caused by an infected decubitus ulcer and ventricular arrhythmia, respectively. Graft-versus-host disease occurred in a 65-year-old woman on the 12th postoperative day, and she died on the 20th postoperative day despite receiving large doses of steroids. A 64year-old woman who died of sepsis had a decubitus ulcer at the onset of an acute dissection because she had been bedridden for 1 year because of an old extended myocardial infarction with congestive heart failure. The ulcer became infected after repair of an aortic dissection, and sepsis occurred after prosthetic valve endocarditis. She died on the 90th postoperative day. A 70-year-old woman who died of ventricular arrhythmia underwent an emergency operation without preoperative angiography. After an aortic arch repair, ventricular fibrillation suddenly occurred after complete atrioventricular block on the first postoperative day. She could not be resuscitated. This event was suspected to have been caused by pre-existing coronary artery disease.

In 2 patients the surgical wound was reopened because of bleeding from the anastomotic site of the carotid artery and distal aortic stump, respectively. All patients regained consciousness without any sign of stroke. Spinal damage was noted in 1 patient who had paraparesis and myonephropathic metabolic syndrome postoperatively. Another 4 patients had ischemic damage of various tissues postoperatively as a result of hypoperfusion either during or after the operation: paralytic ileus in 1 patient, splenic infarction in 1, lower limb ischemia in 1, and renal infarction in 1. Two patients temporarily required hemodialysis after the operation because of acute renal failure, and both of these patients completely recovered from renal failure during their hospitalization. No respiratory failure requiring the aid of a respirator for more than 3 days was observed.

Neurologic complications. Careful neurologic examinations, which included psychometric tests (see the appendix), were conducted by neurologists on the first postoperative day and at the time of discharge. Twenty-six patients, except for 1 who died early after the operation, were available for a neurologic evaluation, and all of them underwent CT of the head. There were no patients in whom new lesions were observed on their CT scan. There were no patients demonstrating either a transient neurologic deficit or seizures.
According to the results of psychometric tests, a change in character was suspected in only 1 patient, but the remainder did not show any memory disturbance or cognitive dysfunction.

Paraparesis was noted in 1 patient. This patient had paraparesis before undergoing an emergency operation, probably because of cord ischemia. The aortic dissection in the descending aorta was considered to be the cause of the obstruction of the blood supply to the spinal cord. The patient with paraparesis did not recover completely after the repair of the aortic dissection, but he could walk by himself at the time of discharge.

The interval between the completion of the operation and regaining consciousness ranged from 0.5 to 13 hours and averaged $4.7 \pm 3.9$ hours. This long interval may be attributable to the use of large doses of thiopental. No other side effects related to thiopental were observed.

Postoperative aortograms and computed tomograms. Except for 2 patients who required hemodialysis postoperatively, all survivors of the operation underwent aortography and body CT $(n=23)$. Using these studies, radiologists evaluated the state of residual false channels. No evidence was observed of persisting false channel in $15(65 \%)$ patients. A false channel persisting in the abdominal aorta, which was perfused through the intimal tear in the distal descending aorta or iliac arteries, was seen in 7 patients. In 1 patient a minor leakage was demonstrated at the distal anastomotic site. His false channel, however, was found to be totally thrombosed on CT 6 months after the operation.

Midterm results. The 24 survivors were followed postoperatively for an average of 2.3 years (range, 5 months to 4 years). All patients were followed up monthly, and the state of the residual false channels was evaluated by CT every 6 months. Two patients underwent reoperation for recurrence of a dissection in the proximal aortic stump. In 1 patient a dissection recurred around the sinus of Valsalva, and acute aortic regurgitation also recurred 1.5 years after the initial operation. In another patient severe hemolysis and deformity of the sinus of Valsalva occurred 7 months after the initial operation. In both patients the intima of the sinus of Valsalva was detached from the aortic wall, and a reconstruction preserving the aortic valve was judged to be difficult. A replacement of the sinus of Valsalva with a Freestyle stentless porcine aortic root bioprosthesis (Medtronic, Inc, Minneapolis, Minn) with coronary artery reimplantation was performed in both patients. One of these patients died during the operation as a result of left ventricular dysfunction. Other than these serious events, there was one transient ischemic attack 


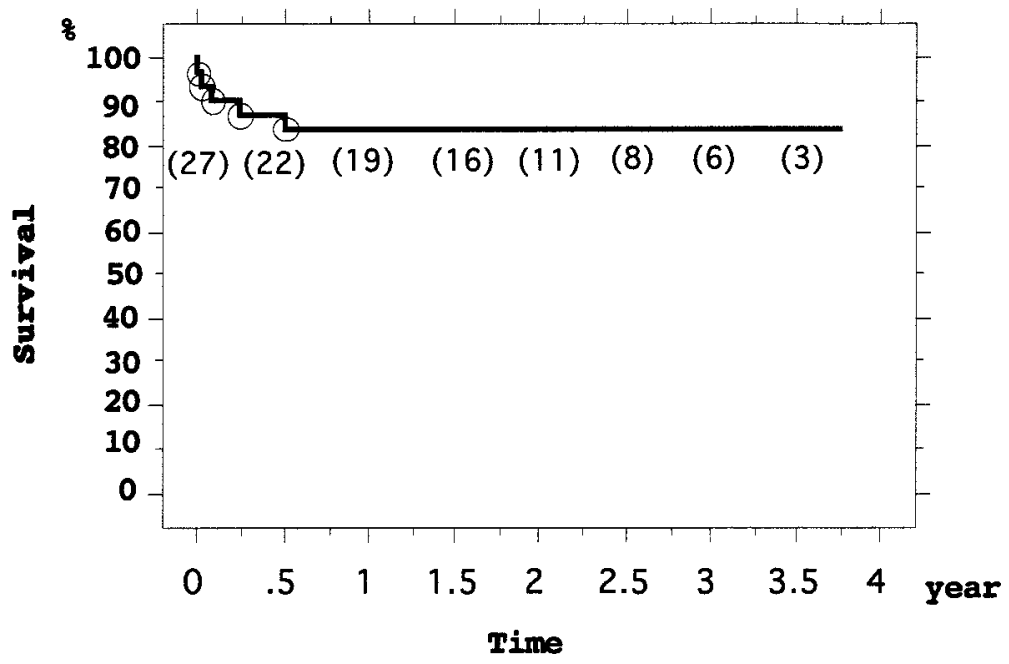

Fig 1. Survival of patients who had acute aortic arch dissection and underwent simultaneous replacement of the ascending and transverse aorta with the prosthesis. Numbers in parentheses indicate the number of patients at risk at each interval. The confidence limits for survival at a half year ranges from $78 \%$ to $92 \%$.

in a patient and upper limb ischemia in another patient caused by the occlusion of the limb graft to the left subclavian artery. Both patients were successfully managed with conservative treatment. Fig 1 shows the actuarial survival curve estimated by the Kaplan-Meier method with a 4-year survival of $85 \%$. Fig 2 shows the freedom from reoperation estimated by the same method, with a 4-year freedom from reoperation of $90 \%$.

\section{Comment}

All 27 patients had an acute type A aortic dissection, and their aortic arch was injured by the dissection. In $19(70 \%)$ of these patients, the intimal tear was located only in the ascending aorta. According to most reports, including the most recent ones, ${ }^{4,5,9,10}$ the patient whose aortic arch was involved by the dissection and in whom no intimal tears were observed in the aortic arch should only undergo a repair of the ascending aorta because the simultaneous repair of the aortic arch increases the difficulty and risk of the operation. In addition, in the large study by Crawford and colleagues, ${ }^{6}$ an extended aortic repair was performed only when the intimal tear was located in the aortic arch, when the dissected aortic arch was ruptured, or when the false channel in the aortic arch was severely dilated. On the other hand, few authors include all patients who had an acute aortic arch dissection and underwent a total aortic arch replacement. Massimo and colleagues ${ }^{11}$ report on 54 patients who underwent an extended and total aortic arch resection. Their 30 -day mortality was $20 \%$, which is not significantly different from that recently reported by others but higher than that reported herein.

Recently, the procedures for cerebral protection have greatly improved. Both selective cerebral perfusion techniques $^{12}$ and retrograde cerebral perfusion techniques $^{13}$ showed excellent results, and we recently reported the protective effects of large doses of thiopental against cerebral global ischemia under profound hypothermic conditions. ${ }^{7}$ In addition to these significant improvements in perioperative management, surgical techniques have also been improved as a result of advances, such as biologic glues ${ }^{14}$ and the introduction of nonwrapping techniques. ${ }^{15}$ In the optimal surgical strategy the dissected aortic arch should be replaced with a prosthetic graft irrespective of whether the intimal tear is located in the aortic arch. As a result, our hospital mortality was only $11 \%$, which is lower than that in most other series, including recent ones, in which an aggressive aortic repair was not tried.9,10,16-18

We consider that total aortic arch replacement for acute aortic arch dissection has several advantages over the ascending aortic replacement alone. All possible intimal tears in the aortic arch can be totally resected, even when the intimal tear is not visible through an aortotomy of the ascending aorta. We have sometimes experienced that intimal tears, which were judged not to exist in the aortic arch during the operation, were later found in the resected aortic arch specimen. A second reason is that the number of patients who might require a late reoperation can be expected to be lower 


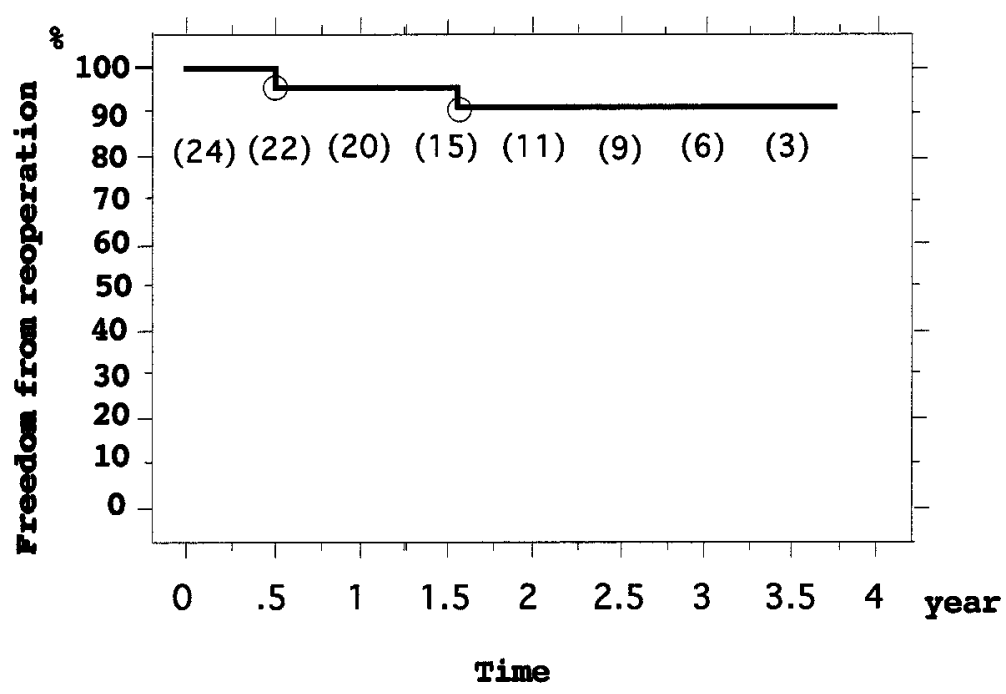

Fig 2. Freedom from reoperation in patients who had acute aortic arch dissection and underwent simultaneous replacement of the ascending and transverse aorta with the prosthesis. Numbers in parentheses indicate the number of patients at risk at each interval. The confidence limits for freedom from reoperation at 1 and 2 years range from $91 \%$ to $100 \%$ and $83 \%$ to $97 \%$, respectively.

than when a dissection remains in the aortic arch. Crawford and colleagues ${ }^{6}$ suggested that including the aortic arch in the resection of the ascending aorta for an acute dissection when the aortic arch contains the dissection resulted in fewer late reoperations. Furthermore, a previous operation involving the ascending aorta has also been reported to be a significant risk factor in patients who require aortic arch repair. ${ }^{19}$ The third reason for including the arch is that the prosthetic graft can be anastomosed to the aortic wall more easily. Generally, it is thought that the nearer to the intimal tear, the more fragile the aortic wall will be. Finally, the area of the aortic wall that had been crossclamped during the operation can be totally resected. The ascending aorta needs to be crossclamped just before the origin of the innominate artery when replacement of the ascending aorta alone is performed without using the hypothermic circulatory arrest. The site of crossclamping is considered a risk of developing another intimal tear postoperatively.

In our study the hospital mortality was $11 \%$, which is lower than that recently reported by other authors for patients with an acute type A aortic dissection. ${ }^{4,5,9,10,18}$ An extended aortic resection thus allows the resection of the intimal tear to be more accurate and the anastomosis of the prosthetic graft to be more exact while reducing the possibility of a new intimal tear. As a result, we believe that patients with an acute type A aortic dissection will have good survival.
An operation for an acute aortic dissection is often performed on an emergency basis in less-experienced centers. In such a setting, a routine repair of both the ascending and transverse aortas is not considered to be preferable to a more standard repair of the ascending aorta alone. Because both skillful management and techniques will be needed to replace the transverse aorta, in less-experienced centers the more limited standard repair (ie, a solely ascending repair) remains the most appropriate approach.

Aortic valve resuspension for acute aortic regurgitation caused by an acute aortic dissection has been demonstrated to be successful in many reports. ${ }^{20,21}$ We attempted to preserve the aortic valve for all patients, except for patients who had a pre-existing dilatation of the aortic sinuses. Ten patients required aortic valve remodeling: 5 of these were performed with mattress suture support, and the other 5 were performed with glue only because gluing produced a solid reconstruction of the proximal aortic stump. Among these 5 patients who underwent aortic valve remodeling with glue only, 2 required a reoperation as a result of the recurrence of the dissection at the sinus of Valsalva. The reoperation was not thought to be attributable to the preservation of the aortic valve but instead was due to inaccurate remodeling of the aortic sinus without any suture support, suggesting the potential for short-term failure of aortic valve remodeling with glue only. 
Regarding operative morbidity, its high rate in our series remains unacceptable. There were 4 patients who received ischemic damage to various tissues postoperatively: paralytic ileus in 1 patient, splenic infarction in 1 , lower limb ischemia in 1 , and renal infarction in 1 . The causes of these operative complications are considered to be due to malperfusion of various tissues during or after the operation. In each of these 4 patients, preoperative or postoperative aortograms showed the arteries to the organs that received ischemic injuries to branch out from the false channel preoperatively but to be almost obliterated on postoperative aortograms. However, no patients showed any symptoms of visceral ischemia at the time of discharge. Avoiding such ischemic injuries caused by malperfusion during $\mathrm{CPB}$ or by the obliteration of the false channel after the operation will require additional study.

\section{Conclusion}

Although there are limitations to our findings because this was not a randomized prospective study, the number of subjects was too small for an accurate assessment of the surgical method, and the follow-up period was too short to evaluate the reoperation-free survival, a resection of both the ascending and transverse aorta, irrespective of whether the intimal tear is located in the aortic arch, may be an acceptable alternative at experienced centers.

\section{REFERENCES}

1. Bachet J, Teodori G, Goudot B, et al. Replacement of the transverse aortic arch during emergency operation for type A acute aortic dissection. Report of 26 cases. J Thorac Cardiovasc Surg 1988;96:876-86.

2. Lansman SL, Raissi S, Ergin MA, Griepp RB. Urgent operation for acute transverse aortic arch dissection. J Thorac Cardiovasc Surg 1989;97:334-41.

3. Graham JM, Stinnett DM. Operative management of acute aortic arch dissection using profound hypothermia and circulatory arrest. Ann Thorac Surg 1987;44:192-8.

4. Kazui T, Kimura N, Yamada O, Komatsu S. Total arch graft replacement in patients with acute type A aortic dissection. Ann Thorac Surg 1994;58:1462-8.

5. Bachet J, Termignon J, Dreyfus G, et al. Aortic dissection: prevalence, cause, and results of late reoperations. J Thorac Cardiovasc Surg 1994;108:199-206.

6. Crawford ES, Kirklin JW, Naftel DC, et al. Surgery for acute dissection of ascending aorta. Should the arch be included? J Thorac Cardiovasc Surg 1992;104:46-59.

7. Hirotani $T$, Kameda $T$, Kumamoto $T$, Shirota S, Yamano M. Protective effect of thiopental against cerebral ischemia during circulatory arrest. Thorac Cardiovasc Surg 1999;47:223-81.
8. Hirotani T, Kameda T, Kato Y, Shirota S, Fujiwara H. Clinical results of surgery for type A acute aortic dissection using gelatinresorcin-formaldehyde glue. Ann Thorac Cardiovasc Surg 1997;3:395-9.

9. David TE, Armstrong S, Ivanov J, Barnard S. Surgery for acute type A aortic dissection. Ann Thorac Surg 1999;67:1999-2001.

10. Bachet J, Goudot B, Dreyfus GD, et al. Surgery for acute type A aortic dissection: the Hospital Foch experience (1977-1998). Ann Thorac Surg 1999;67:2006-9.

11. Massimo CG, Presenti LF, Marranci P, et al. Extended and total aortic resection in the surgical treatment of acute type A aortic dissection: experience with 54 patients. Ann Thorac Surg 1988;46:420-4.

12. Kazui T, Kimura N, Yamada O, Komatsu S. Surgical outcome of aortic arch aneurysms using selective cerebral perfusion. Ann Thorac Surg 1994;57:904-11.

13. Ueda Y, Okita Y, Aomi S, Koyanagi H, Takamoto S. Retrograde cerebral perfusion for aortic arch surgery: analysis of risk factors. Ann Thorac Surg 1999;67:1879-82.

14. Guilmet D, Bachet J, Goudot B, et al. Use of biological glue in acute aortic dissection: preliminary clinical results with a new surgical technique. J Thorac Cardiovasc Surg 1979;77:516-21.

15. Massimo CG, Presenti LF, Favi PP, et al. Excision of the aortic wall in the surgical treatment of acute type-A aortic dissection. Ann Thorac Surg 1990;50:274-6.

16. Miller DC, Stinson EB, Oyer PE, et al. Operative treatment of aortic dissections: experience with 125 patients over a sixteenyear period. J Thorac Cardiovasc Surg 1979;78:365-82.

17. Ergin MA, Phillips RA, Galla JD, et al. Significance of distal false lumen after type A dissection repair. Ann Thorac Surg 1994;57:820-5.

18. Hagan PG, Nienaber CA, Isselbacher EM, et al. The international registry of acute aortic dissection (IRAD): new insights into an old disease. JAMA 2000;283:897-903.

19. Crawford ES, Svensson LG, Coselli JS, Safi HJ, Hess KR. Surgical treatment of aneurysm and/or dissection of the ascending aorta, transverse aortic arch, and ascending aorta and transverse aortic arch: factors influencing survival in 717 patients. J Thorac Cardiovasc Surg 1989;98:659-74.

20. Jex RK, Schaff HV, Piehler JM, et al. Repair of ascending aortic dissection: influence of associated aortic valve insufficiency on early and late results. J Thorac Cardiovasc Surg 1987;93:375-84.

21. Mazzucotelli JP, Deleuze PH, Baufreton C, et al. Preservation of the aortic valve in acute aortic dissection: long-term echocardiographic assessment and clinical outcome. Ann Thorac Surg 1993;55:1513-7.

\section{Appendix}

The Hasegawa dementia score was used to detect a decline in neurocognitive function. This examination is widely used in Japan. Scores (range, 0-30) of less than 24 on this examination are indicative of cognitive dysfunction. This examination was performed on the 14th postoperative day. The individual change score could not be analyzed because of the preoperative critical state of patients. The Hasegawa dementia score measured postoperatively averaged $27.0 \pm 1.8$. 